\title{
Exploring Privacy Aspects of Smartphone Notifications
}

\author{
Priyanka Verma* \\ vpriyanka0492@gmail.com \\ Birla Institute of Technology and Science (BITS) Pilani \\ Pilani, India
}

\author{
Sameer Patil \\ patil@indiana.edu \\ Luddy School of Informatics, Computing, and Engineering, \\ Indiana University Bloomington \\ Bloomington, Indiana, USA
}

\begin{abstract}
Notifications are one of the most important features of mobile devices. Users receive multitudes of notifications every day. These notifications often carry sensitive content such as private messages, financial information, authentication tokens, etc. To understand privacy considerations related to notification content and delivery, we conducted a study in which participants $(n=206)$ described their preferences and practices regarding smartphone notifications. A majority of the participants $(61 \%)$ reported at least one negative experience connected to notifications. We report on various privacy violations that arise due to notifications, such as unwanted information disclosure, intimacy breach, and inopportune intrusion. Our work contributes to a better understanding of privacy risks presented by notifications on mobile devices and points to several design suggestions for privacy-sensitive notification delivery mechanisms.
\end{abstract}

\section{CCS CONCEPTS}

- Human-centered computing $\rightarrow$ Smartphones; Mobile devices; Empirical studies in ubiquitous and mobile computing; $\bullet$ Security and privacy $\rightarrow$ Social aspects of security and privacy; Usability in security and privacy.

\section{KEYWORDS}

smartphone notifications, privacy, information disclosure, device sharing, intrusion

\section{ACM Reference Format:}

Priyanka Verma and Sameer Patil. 2021. Exploring Privacy Aspects of Smartphone Notifications. In 23rd International Conference on Mobile HumanComputer Interaction (MobileHCI '21), September 27-October 1, 2021, Toulouse \& Virtual, France. ACM, New York, NY, USA, 13 pages. https://doi.org/10. $1145 / 3447526.3472065$

\section{INTRODUCTION}

Notifications are an important method of information delivery on mobile devices. The device operating system as well as third party

\footnotetext{
"The work described in the paper was done while the author was a research scholar at the Luddy School of Informatics, Computing, and Engineering, Indiana University Bloomington, USA.
}

This work is licensed under a Creative Commons

Attribution-NonCommercial-ShareAlike International 4.0 License.

MobileHCI '21, September 27-October 1, 2021, Toulouse \& Virtual, France

(c) 2021 Copyright held by the owner/author(s).

ACM ISBN 978-1-4503-8328-8/21/09.

https://doi.org/10.1145/3447526.3472065 apps generate notifications to alert users about a number of matters, such as previews of incoming content, reminders for upcoming events and tasks, availability of software updates, warnings regarding data access, and so on. Smartphone users receive more than 50 notifications per day on average, making notifications more common than phone calls [36, 37].

While the information contained in notifications is useful, it can often be privacy sensitive. For instance, notifications may contain personally identifiable information or confidential matters that users do not wish to divulge to others. Since notifications are typically triggered by the actions of other parties, users cannot predict when a notification may appear. If notifications containing private information are displayed when a user is in a situation in which the mobile device is shared with another person or the device screen is easily viewable by other parties, the user may experience privacy violations owing to undesirable information disclosure. Moreover, notifications that interrupt the user at inopportune moments can violate privacy by intruding upon solitude, a facet of privacy [40].

Although unwanted information disclosures and associated privacy concerns have been investigated in the specific context of email notifications [21], there has not yet been a systematic and comprehensive investigation of privacy aspects of notifications on mobile devices in general. We aim to fill this gap by investigating user preferences and practices regarding notifications received via mobile devices from a privacy point of view. Specifically, we address the following research questions:

- RQ1: What are the privacy considerations associated with notifications received on mobile devices?

- RQ2: How are the privacy considerations associated with notifications influenced by contextual factors?

- RQ3: How do device mechanisms to control notifications (e.g., silent mode, notification delivery preferences, etc.) pertain to privacy aspects related to notifications?

We sought to answer the above questions using an online questionnaire administered via the Amazon Mechanical Turk (AMT) platform. The questionnaire covered device settings, device-sharing behavior, notification content, and contextual aspects related to notification delivery and reception. Based on questionnaire responses of 206 participants, we found that notifications on mobile devices can indeed impact the privacy of the recipients and those whose information is included in the notification. We further uncovered that privacy threats of notifications are particularly high in situations that involve device sharing. The contribution of our work lies in complementing prior research on notifications and extending it to the context of mobile devices. Our findings provide an improved understanding of privacy aspects connected to mobile device notifications, and the insight can be applied to address privacy concerns and avoid privacy violations. 
In the following sections, we describe notification delivery mechanisms typical of current smartphones, provide an overview of notification customization mechanisms, and explain the method used to conduct the study. We then present the findings of the study and apply them to propose privacy-enhancing improvements to the delivery of notifications on mobile devices.

\section{RELATED WORK}

Our study lies at the intersection of individual privacy concerns, privacy issues specific to mobile devices, and privacy aspects connected to notifications. Below we cover the salient work in each space.

\subsection{Individual Privacy Concerns}

Altman stated that different aspects of individual privacy concerns are connected to the "interplay of people, their social world, the physical environment, and the temporal nature of social phenomena" [5]. As a result of its contextual and nuanced nature, privacy lacks a universal definition and has been conceptualized in a number of ways. For instance, Westin's [47] influential characterization describes privacy as being related to four states: Solitude, Intimacy, Anonymity, and Reserve. As summarized by Margulis [25], Solitude is being free from observation by others; Intimacy refers to small group seclusion to achieve close, relaxed, frank relationships among group members; Anonymity provides freedom from identification and from surveillance in public places and for public acts; and Reserve is based on the desire to limit disclosures to others. Solove [40] has described how everyday activities are connected to conceptual characterizations of privacy such as "intrusion" of solitude: "intrusion involves invasions or incursions into one's life. It disturbs the victim's daily activities, alters her routines, destroys her solitude, and often makes her feel uncomfortable and uneasy" (p. 549). Digital intrusion, such as spam, telemarketing, unwanted or inopportune notifications, etc., violate privacy by disrupting solitude, interrupting ongoing activities, and creating discomfort, much the same as intrusion experienced in physical spaces [40]. Our study examines how notification content and delivery are connected to these various facets of privacy.

To measure individual privacy concerns in the digital context, researchers have developed standard instruments pertaining to information privacy, such as the Concern for Information Privacy (CFIP) [39] scale, later refined to the Internet Users' Information Privacy Concerns (IUIPC) scale [24]. Specifically in the realm of technology-mediated interpersonal interactions, researchers have been studying privacy preferences and practices through the lenses of information disclosure (e.g., [31]) and self presentation (e.g., [23]), covering a variety of applications, such as workplace collaboration (e.g., [32]), messaging platforms (e.g., [22]), location-sharing systems (e.g., [33]), social media (e.g., [48]), etc. The findings of these investigations provide empirical confirmation of the contextual variation in privacy preferences and practices. Privacy violations are often a result of violations of such contextual needs and expectations [30]. Therefore, our study examines how privacy aspects connected to notifications might be influenced by contextual factors, such as time and place of delivery, type of app, etc.

\subsection{Privacy in Mobile Devices}

As mobile devices have become an integral part of everyday life, Human-Computer Interaction (HCI) researchers have focused on understanding privacy considerations pertaining specifically to mobile devices. For instance, to help researchers measure individual privacy concerns specifically in the context of smartphone apps, $\mathrm{Xu}$ et al. [49] developed the Mobile Users' Information Privacy Concerns (MUIPC) scale containing the constructs of Perceived Surveillance and Perceived Intrusion experienced when using mobile devices and apps. In addition, researchers have proposed a number of enhancements to support user privacy needs and expectations when using mobile devices and applications. For instance, researchers have developed techniques to raise user awareness regarding privacy-affecting operations of devices and apps (e.g., [9]) and proposed "soft paternalistic" [8] approaches that nudge users to make privacy-protecting decisions $[4,7,28]$.

In a complimentary vein, researchers have explored more effective mechanisms for device locking, such as context-sensitive screen locks [29] and progressive authentication [38]. Further, tools such as ColorSnakes [18] and XSide [14] protect authentication input from the threat of shoulder surfing [15]. Relatedly, Von Zezschwitz et al. [42] designed a photo-obfuscation technique to prevent privacy leaks when jointly browsing photo albums on a smartphone in order to locate and show specific photos to others, and Ali et al. [2] developed the iAlert tool to alert users to the presence of bystanders who might be able to view the device screen.

However, none of these efforts have focused explicitly on the notification features of mobile devices. Moreover, the various tools and techniques mentioned above do not take into account device access by others (except bystanders), as is the case when sharing the device. Yet, device sharing is a common practice [26] (especially in less individualistic societies [1]) that has been found to impinge upon privacy $[19,20]$. Our study aims to shed light on privacy concerns with notifications received while sharing the device with others.

\subsection{Privacy Aspects of Notifications}

Notification mechanisms have been a feature of operating systems and applications for several decades. While researchers have paid particular attention to the interruptions caused by notifications, these investigations have been concerned primarily with measuring and avoiding the negative impact of interruptions on productivity and affect. To that end, such efforts attempt to identify opportune moments when a user might be interruptible and receptive to the notification (e.g., [12, 17, 27, 34, 50]). Apart from the disruption caused by interruptions, privacy aspects of notifications are still relatively unexplored. Vardhan et al. [41] designed a classifier to determine whether a notification is likely to be private based on the title, content, and the name of the application that generated the notification. The classifier can be adjusted based on user feedback regarding which notifications should be treated as private. However, the classifier does not take into account the impact of contextual factors, such as time, place, delivery mode, etc. A recent study by Kim et al. [21] found that the context in which notifications are received on a mobile device does impact the likelihood and severity of undesired disclosure of private information. However, the study 
was narrowly scoped because it included a sample from a single organization and considered only email notifications in the context of in-person workplace meetings. Our study builds on the works of Vardhan et al. [41] and Kim et al. [21] by broadening the scope to cover multiple facets of privacy aspects connected to all types of notifications received on mobile devices in a diverse variety of contexts. Moreover, our study includes consideration for the privacy of other parties whose information might be contained within the notification content.

\section{METHOD}

We designed a questionnaire to address our research questions. All study materials and procedures (see Supplementary Material) were approved by our institution's Institutional Review Board (IRB). The following subsections describe the design and deployment of the study, provide an overview of the sample, and specify our data analysis approach.

\subsection{Questionnaire Design}

We designed a questionnaire that asked smartphone users about their preferences and practices related to notifications received on their devices. Apart from inquiring about device characteristics and preference settings related to notifications, we included questions regarding the context in which notifications were received and read and practices related to sharing devices with others. To delve deeper into these aspects via a tighter coupling between general preferences and practices and the specifics of particular notifications, we asked questions pertaining to the latest notification, such as delivery mode, context, privacy concern, etc.

Since privacy violations are negative experiences, we further asked participants to elaborate on their most negative experiences connected to notifications. Such an approach allowed us to capture a broad set of negative experiences without explicitly priming and constraining participants by using the term 'privacy.' We then asked for the level of concern for someone other than the participant reading notification content for a variety of common app types (i.e., Instant messaging, Social media, Calendar, E-mail, Banking and payments, Health and fitness, and Dating).

Following the above core questions related to our research questions, we used standard scales from the literature as measures of baseline privacy concern when using mobile devices (the Perceived Intrusion and Perceived Surveillance subscales of MUIPC [49]) and general technical expertise (General Digital Difficulties subscale of the Digital Difficulties scale [6]). At the end of the questionnaire, we collected standard demographic information. Wherever applicable, we provided the option to enter open-ended explanations.

We tested and iteratively refined the questionnaire via multiple small-scale pilots involving students and postdoctoral researchers at our institutions as well as personal contacts of the authors. The pilots served to ensure that the questions were easily comprehensible and the questionnaire operation was error-free.

\subsection{Study Deployment}

We deployed the study as a Human Intelligence Task (HIT) available on the AMT crowdwork platform between November 12, 2020 and December 1, 2020. Since privacy is known to vary by culture, we restricted participation to those from the United States to limit the impact of the answers being affected by cultural variation. To maximize the chances of receiving high-quality responses, we further restricted participation to those with a task approval rating of $95 \%$ or higher and at least 50 completed tasks.

Upon accepting the HIT, participants received a link to the online questionnaire implemented via the Qualtrics platform. After reading information about the study and consenting to participate, participants proceeded to the questionnaire that began with a question to gauge commitment to providing thoughtful answers [3]. No questions were mandatory; participants could choose to skip any question they did not wish to answer. At the end of the questionnaire, we provided participants with a randomly-generated unique code to be entered on AMT as proof of completion. All those who answered the questionnaire attentively and entered a valid code were compensated US $\$ 1.80$ for their participation. Based on a mean completion time of 19 minutes, the compensation translates to roughly $\$ 6 /$ hour, which is typical of AMT based studies.

\subsection{Sample Characteristics}

We obtained responses from individuals covering a broad age range from 18 to $70($ median $=35$; mean $=36)$. Most $(88 \% ; n=183)$ reported living the United States for more than 10 years. Participants were split $61 \%(n=126)$ and $38 \%(n=79)$ between men and women respectively, with two participants choosing not to report gender. While nearly three quarters of the participants were White Caucasians $(75 \% ; n=154)$, the remainder of the sample included individuals from a variety of ethnic backgrounds: American Indian or Native American $(1.5 \% ; n=3)$, Asian $(6.8 \% ; n=14)$, Black or African American (10.7\%; $n=22)$, Hispanic (3.4\%; $n=7)$, and Native Hawaiian or Pacific Islander $(1.5 \% ; n=3)$. One participant reported being multi-ethnic, and two chose not to report ethnicity. The participants reported a wide variety of professional backgrounds, such as construction, management, information technology, media production, art, pharmaceutical, etc. Nearly all participants $(93 \% ; n=192)$ reported at least some college education, with $78 \%(n=165)$ having completed a Bachelor's degree or higher. A majority of the participants were married $(62 \%, n=128)$, while roughly one third were single $(32 \% ; n=66)$; the remaining $6 \%(n=14)$ were separated, divorced, widowed, or did not specify marital status. Most participants $(72 \% ; n=146)$ lived in households of three or more people, with the remaining either living alone $(16 \% ; n=33)$ or with one other person $(13 \% ; n=27)$.

\subsection{Data Analysis}

We first examined the data to flag and filter invalid responses. Of the 275 total responses, we excluded 24 because of duplicate IP addresses. We then filtered out four additional responses that originated from IP addresses outside the United States. We removed an additional 41 responses for failing any of the attention checks embedded within the questionnaire and/or not committing to providing thoughtful answers and/or having a device operating system other than Android or iOS. After applying all filters, we were left with 206 complete and valid responses that we analyzed to derive our findings. 
Given the exploratory nature of our study, we used relevant descriptive statistics for examining the quantitative variables included in the questionnaire. Where applicable, we used inferential statistics to examine the statistical significance of any observations connected to numeric variables. Since the data was not normally distributed, we used non-parametric tests, such as the Kruskal-Wallis test, Mann-Whitney U test, and Wilcoxon signed-rank test, with Bonferroni correction for repeated testing where applicable.

We analyzed the qualitative data collected via open-ended questions by employing three independent coders, one of whom was the first author of this paper. The first author generated an initial list of codes based on a detailed examination of the responses and consultation with the second author. The three independent coders then used the initial list of codes to code all open-ended responses using thematic analysis techniques [10]. During the independent coding process, coders were free to suggest refinements to the existing codes and add codes to the initial list. After the initial independent coding, we consolidated the three sets of codes, flagged disagreements, and held a discussion among the three coders to resolve the discrepancies. The discussion resulted in renaming some of the initial codes and adding a few new ones, culminating in full agreement among the three coders. We then clustered similar codes into higher-level themes connected to concepts taken from the literature [40, 47] (see Section 2.1).

\section{FINDINGS}

We analyzed the questionnaire responses to investigate privacy aspects connected to notifications (RQ1). Next, we examined how privacy considerations vary based on content from different types of apps and the contexts in which others parties can view the device screen (RQ2). We then explored the interplay between privacy concerns and device-control mechanisms and technical expertise (RQ3).

\subsection{Privacy Aspects Connected to Notifications (RQ1)}

We studied privacy considerations related to notifications as part of negative experiences with notifications on mobile devices. A majority of the participants $(61 \% ; n=128)$ had encountered at least one negative experience connected to smartphone notifications (see Figure 1). A little more than half of the participants $(51 \% ; n=106)$ reported 1-5 instances of negative experiences. We performed a Mann-Whitney U Test to examine whether negative experiences connected to notifications are associated with the device operating system, with number of negative experiences as the dependent variable and type of operating system as the independent variable. We found no statistically significant differences in the number of negative experiences between users of Android and iOS $(p=0.92)$.

To understand how negative experiences relate to privacy violations, we analyzed the 91 open-ended responses in which participants elaborated on their most negative experiences. Table 1 presents the four salient themes that emerged from the responses: (i) unintended information disclosure; (ii) privacy breaches during shared use of the device; (iii) intrusion in task, solitude, or digital space; and (iv) invasion of social intimacy.

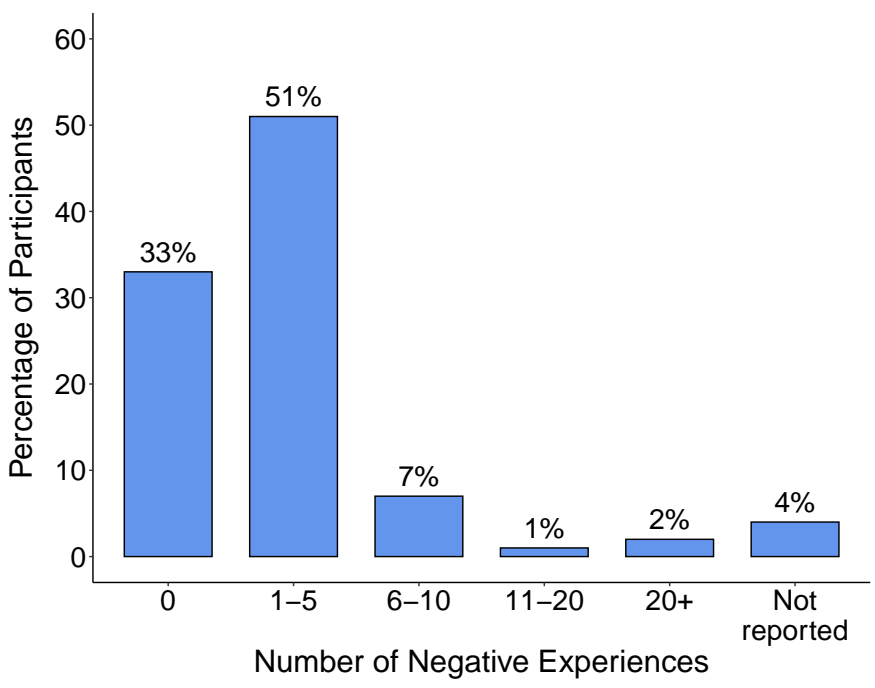

Figure 1: Distribution of participants based on the reported number of negative experiences connected to notifications.

4.1.1 Unwanted information disclosure connected to notifications. We found that smartphone notifications deliver and disclose private content $(n=21)$, information about other parties $(n=2)$, and inappropriate content $(n=8)$. Inappropriate content refers to racy photos or videos, adult language, etc.

"I was texting something personal with my hubby, suddenly when I was with my colleague it [notification] popped up and the person read it." (P71)

"I received a text from someone whom I wasn't supposed to be messaging, and my friend who was with me at that point saw the text and whom it was from pop up on my phone." (P38)

As some of the example comments in Table 1 indicate, such unwanted information disclosure can lead to misunderstandings $(n=$ 4) and conflicts $(n=5)$.

To identify the prevalence of the risks of unwanted disclosure of private information, we analyzed the responses pertaining to the latest notification received by the participants. These notifications were generated by apps from a wide variety of categories, such as social media, messaging, news, shopping, ridesharing, dating, and others. A majority of the participants $(52 \% ; n=109)$ reported that they would be concerned (i.e., somewhat concerned or extremely concerned on a five-point scale) if someone other than them viewed the content of the latest notification (see Figure 3a).

Next, we examined whether privacy concern for notification content differs across app categories and audiences. We found that content from different types of apps is associated with different levels of privacy concerns when seen by a family member or a colleague (see Figure 2 and Table 2). Wilcoxon signed-rank tests with Bonferroni correction confirmed that the privacy concern regarding family members is statistically significantly different from that for colleagues $(p<0.05)$ for all app categories except Dating. Overall, people are more concerned about notifications leaking information to colleagues than to family members. Within each of these two 
Table 1: Categories of privacy violations connected to notifications reported by the participants.

\begin{tabular}{|c|c|c|c|}
\hline Theme & Code & Count & Example Comments \\
\hline \multirow{3}{*}{$\begin{array}{l}\text { Unintended } \\
\text { information } \\
\text { disclosure }\end{array}$} & $\begin{array}{l}\text { Private content } \\
\text { disclosed }\end{array}$ & 21 & $\begin{array}{l}\text { "My girlfriend saw a message from one of my friends that } \\
\text { was too private for her concern." (P93) }\end{array}$ \\
\hline & $\begin{array}{l}\text { Inappropriate } \\
\text { content disclosed }\end{array}$ & 7 & $\begin{array}{l}\text { "Someone sent me inappropriate photos without permission } \\
\text { and my spouse saw them. She thought I was cheating." (P104) }\end{array}$ \\
\hline & $\begin{array}{l}\text { Content about third } \\
\text { parties disclosed }\end{array}$ & 2 & $\begin{array}{l}\text { "One of my friends read a message that was about her, and } \\
\text { it made her upset." (P46) }\end{array}$ \\
\hline \multirow[t]{2}{*}{ Device sharing } & $\begin{array}{l}\text { Device with another } \\
\text { party }\end{array}$ & 7 & $\begin{array}{l}\text { "When my phone was with my sibling, my partner sent me } \\
\text { a romantic message. My sister saw that message." (P139) }\end{array}$ \\
\hline & $\begin{array}{l}\text { Device screen projected } \\
\text { on a public display }\end{array}$ & 2 & $\begin{array}{l}\text { "I was working on something for work where I had to cast } \\
\text { my device screen for others to see. The notification was } \\
\text { highly personal." (P44) }\end{array}$ \\
\hline \multirow[t]{5}{*}{ Intrusion } & $\begin{array}{l}\text { Digital intrusion (with } \\
\text { undesired content, mal- } \\
\text { ware, etc.) }\end{array}$ & 23 & $\begin{array}{l}\text { "One time, I clicked the link in the notification without study- } \\
\text { ing it in detail, resulting in the phone being infected with a } \\
\text { virus." (P41) }\end{array}$ \\
\hline & Intrusion in solitude & 4 & $\begin{array}{l}\text { "The most negative experience was being woken up by noti- } \\
\text { fications in the middle of the night and not being able to fall } \\
\text { back to sleep even though I had work in the morning." (P89) }\end{array}$ \\
\hline & Unease or discomfort & 8 & $\begin{array}{l}\text { "I kept on getting advertisement notifications, repeatedly } \\
\text { one after another, from a shopping app I had that were really } \\
\text { not related to the app itself, and [the notifications] would } \\
\text { not stop unless I would open the app right away. So I wound } \\
\text { up deleting the app altogether." (P138) }\end{array}$ \\
\hline & Distraction & 5 & $\begin{array}{l}\text { "Group chats are always annoying and they send a lot of } \\
\text { notifications. It is distracting and irritating especially when } \\
\text { you're busy." (P79) }\end{array}$ \\
\hline & Frequent interruption & 14 & $\begin{array}{l}\text { "Sometimes my friend likes to blow up iMessage and I get } \\
\text { irritated or annoyed." (P147) }\end{array}$ \\
\hline \multirow[t]{3}{*}{ Intimacy breach } & $\begin{array}{l}\text { Interruption in a social } \\
\text { setting }\end{array}$ & 5 & $\begin{array}{l}\text { "I received an email from a colleague that popped up in my } \\
\text { notifications when I had my sound on and was attending a } \\
\text { formal dinner party, and it went off during a speech." (P86) }\end{array}$ \\
\hline & Misunderstanding & 4 & $\begin{array}{l}\text { "I had an ex get jealous because he saw a text from an un- } \\
\text { known number. He accused me of cheating when it was not } \\
\text { the case." (P18) }\end{array}$ \\
\hline & Conflict & 5 & $\begin{array}{l}\text { "Someone saw a personal text for me and got mad at me" } \\
\text { (P125) }\end{array}$ \\
\hline
\end{tabular}

audience types, people deem notifications related to Banking and payments as the most sensitive and those regarding Calendar information as the least sensitive. Sensitivity of notifications from apps that involve interaction with other parties (i.e., Dating, E-mail, Instant messaging, and Social media) falls just below that for Banking and payments.

We then sought to identify the prevalence of the risk of disclosure of private information of other parties. Out of the 206 participants, 84 mentioned that the last notification they received contained information pertaining to another party. Around 70\% ( $n=59$ of these 84 participants reported that the other person would be concerned (i.e., somewhat concerned or extremely concerned on a five-point scale) if someone other than the intended receiver saw the notification content (see Figure 3b). Overall, there was a risk of unwanted disclosure of private information for other parties reported by about one-third of the entire sample.

4.1.2 Privacy invasion connected to notifications. Participant responses indicate that notifications are connected to the facet of privacy linked to intrusion [40] because they disrupt ongoing tasks/activities $(n=5)$, interrupt frequently $(n=7)$, disturb solitude $(n=6)$, and invade digital space $(n=14)$. Notifications additionally impact the privacy facet connected to the intimacy of social settings $(n=6)$ [47].

We characterize 'invasion of digital space' as notifications that contain unwanted content like scam links, fraudulent messages, viruses, and spam (see Section 2.1). For example, a participant complained, "some unwanted video notifications irritate me a lot of 


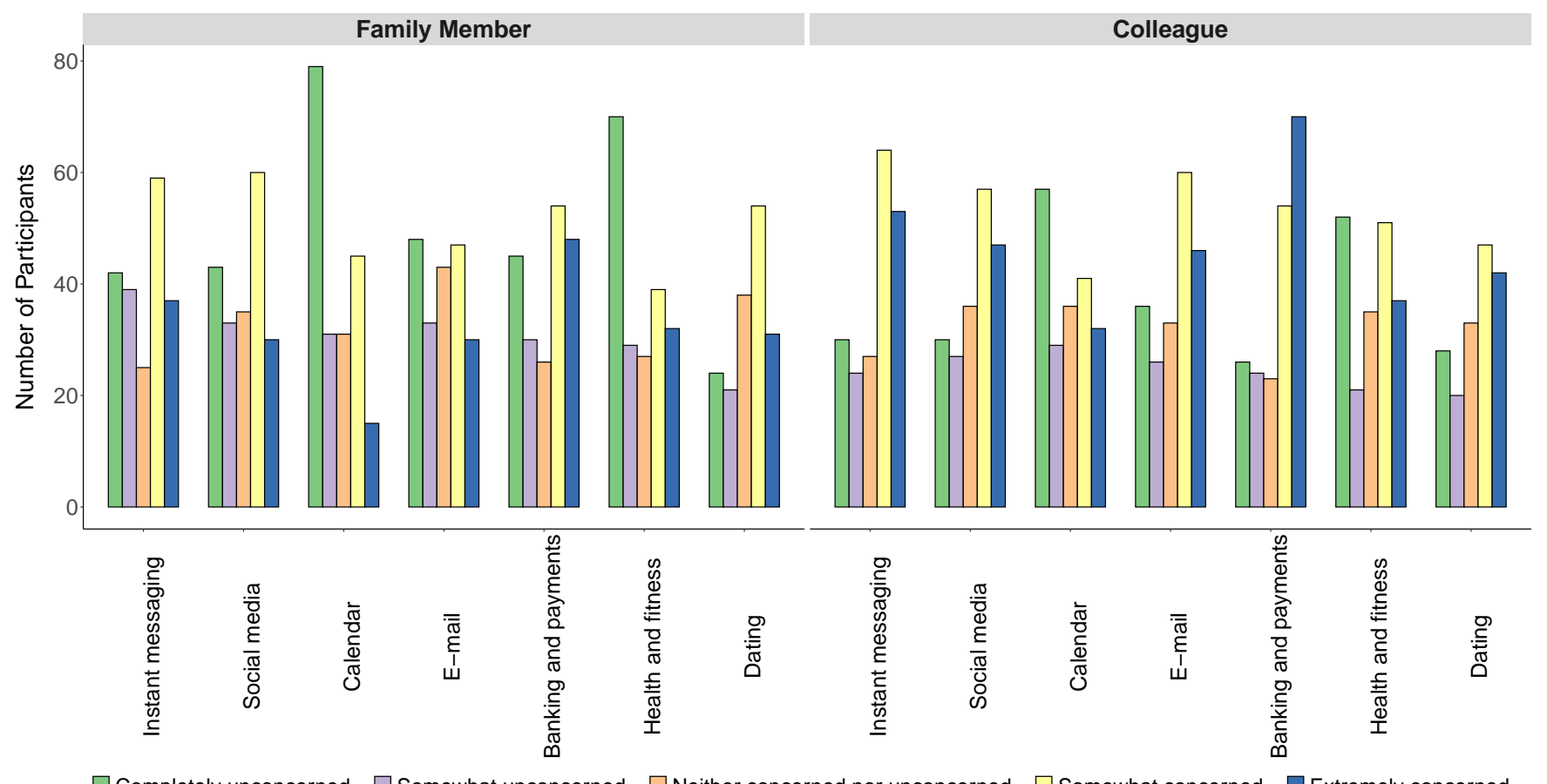

Figure 2: Levels of privacy concern for notification content from various categories of apps read by a family member or a colleague.

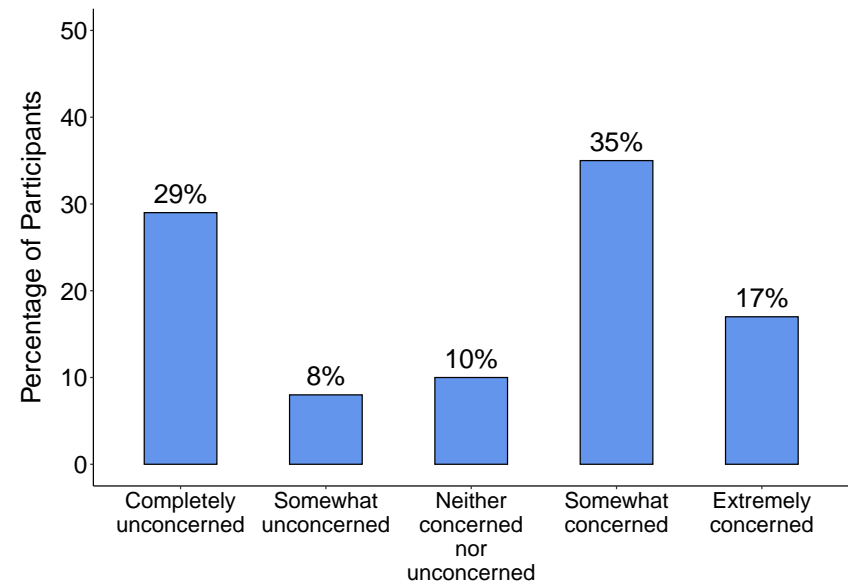

(a) Concern for self.

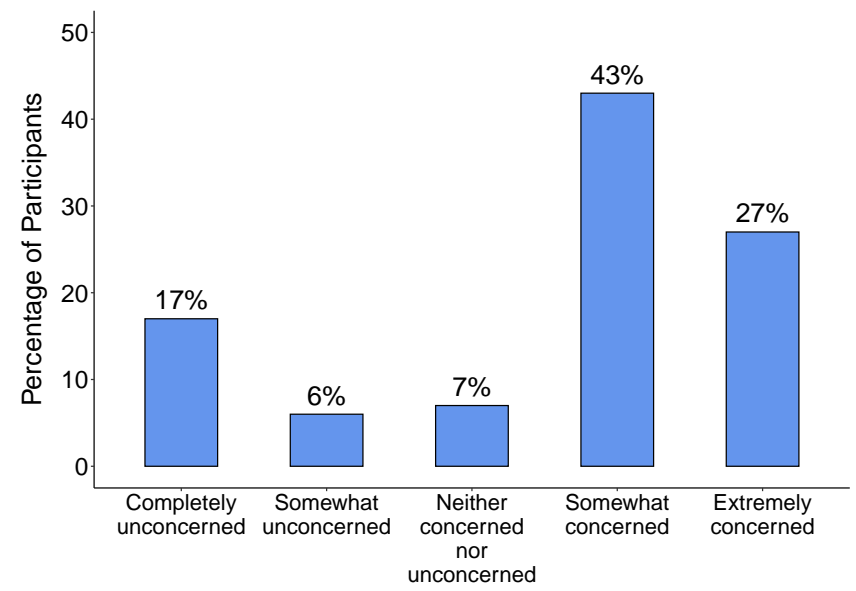

(b) Concern of other parties.

Figure 3: (a) Level of concern for someone other than the participant viewing the latest notification received. (b) Perception regarding the level of concern of other parties if information about that party contained in the latest notification was viewed by someone other than the participant (i.e., the notification receiver).

times" (P52). In addition, many participants reported that notifications caused unwanted interruptions in social settings:

"It was a Facebook notification. I was in a work meeting that was very important and I had forgotten to silence my notifications. It was very embarrassing." (P165)

"notification ring in office meeting." (P206)
Further, several participants mentioned that notifications led to conflict and misunderstanding, thus breaching the Intimacy facet of privacy [47] (see Section 2.1).

Table 3 shows the mean scores for the Perceived Intrusion and Perceived Surveillance subscales from the MUIPC scale [49], grouped by the number of negative experiences connected to notifications reported by the participants. We found the number of negative 
Table 2: Mean level of privacy concern for notification content from various categories of apps read by a family member or a colleague on a five-point scale ranging from $1=\mathrm{Com}$ pletely unconcerned to 5 = Extremely concerned.

\begin{tabular}{lcccccc}
\hline & \multicolumn{2}{c}{$\begin{array}{c}\text { Family } \\
\text { Member }\end{array}$} & & \multicolumn{2}{c}{ Colleague } \\
\cline { 2 - 3 } \cline { 5 - 6 } \multicolumn{1}{c}{ App Categories } & Mean & $S D$ & & Mean & $S D$ \\
\hline Instant messaging & 3.28 & 1.40 & & 3.48 & 1.38 \\
Social media & 3.27 & 1.29 & & 3.39 & 1.30 \\
Calendar & 2.65 & 1.38 & & 2.92 & 1.43 \\
E-mail & 3.17 & 1.32 & & 3.46 & 1.30 \\
Banking and payments & 3.41 & 1.41 & & 3.60 & 1.40 \\
Health and fitness & 2.85 & 1.49 & & 3.09 & 1.43 \\
Dating & 3.35 & 1.30 & & 3.36 & 1.39 \\
\hline
\end{tabular}

experiences to be statistically significantly correlated with Perceived Intrusion (Spearman $\rho=0.17 ; p=0.02$ ) as well as Perceived Surveillance (Spearman $\rho=0.15 ; p=0.03$ ). These positive correlations indicate that those with higher privacy concern when using mobile devices exhibit similarly higher privacy concerns connected to notifications received on those devices.

\subsection{Privacy Considerations due to Contextual Factors (RQ2)}

Close to half of the participants $(46 \% ; n=94)$ reported that they shared their smartphone with their spouse at least once a day (see Figure 4a). Nearly a third of the participants shared their smartphone at least once a day with their siblings $(31 \% ; n=64)$ or friends $(29 \% ; n=59)$. A bit more than a third did so with their parents $(33 \% ; n=67)$ and children above 13 years of age $(35 \% ; n=73)$, and slightly more than a quarter with colleagues $(27 \% ; n=55)$ or even strangers $(25 \% ; n=51)$. Apart from device sharing, half of the participants $(50 \% ; n=102)$ reported that others can view the contents of their notifications at least once a day at home as well as in a variety of other contexts, such as meetings $(27 \% ; n=55)$, classes $(30 \% ; n=62)$, seminars and presentations $(28 \% ; n=57)$, cafeterias and restaurants $(29 \% ; n=59)$, social gatherings $(28 \%$; $n=57)$, stores and markets $(30 \% ; n=62)$, and public transportation $(25 \% ; n=51)$ (see Figure $4 \mathrm{~b})$.

Privacy concerns and risks are exacerbated by the prevalence of the various shared contexts, such as lending the device to someone, viewing information on a device jointly with other parties, screencasting, and presence of bystanders in social and professional settings. Indeed, participants reported several privacy concerns arising from device sharing or visibility of the device screen to someone other than themselves:

"I was watching a video with a younger cousin and the notification popped up. It was adult language that a child (cousin) was able to read." (P76)

"Before giving my device to my sibling, I forgot to turn off the notifications from my personal chat." (P157)
When sharing a device, users have the ability to take a few privacy-protective actions, such as clearing notifications or closing applications. Therefore, we investigated practices and preferences when sharing a device with others (see Figure 5). We found that few participants showed the inclination to use the available privacy-protecting mechanisms; about $39 \%(n=80)$ preferred to clear notifications before handing their devices over to others, a third $(33 \% ; n=68)$ reported closing active applications, and only $10 \%(n=21)$ mentioned using the 'guest mode.' We further analyzed the preferences of those participants who reported that they cleared notifications before handing their devices over to others (see Figure 6). Notably, just $41 \%(n=33)$ of these 80 participants reported always clearing notifications before handing their devices over to a stranger.

\subsection{Relationship between Privacy and Use of Device-State Controls (RQ3)}

Participants reported managing notification delivery via various smartphone mechanisms used routinely to control the device state: Do Not Disturb (DND) mode $(52.4 \% ; n=108)$, silent mode $(79.6 \%$; $n=164)$, turning the device off $(42.2 \% ; n=87)$, airplane mode $(41.2 \% ; n=85)$, and notification management apps from third parties $(60.2 \% ; n=124)$. Table 4 provides the mean usage (in hours) of these mechanisms in a typical day. Based on usage per day, we found that people prefer to use the silent mode and notification management apps over other alternatives to manage notifications. A total of 53 participants provided reasons for controlling device state through the above-mentioned mechanisms (see Table 5). It is interesting to note that participants found the device features helpful to limit intrusion in personal space (e.g., while working or sleeping) and social settings (i.e., in the presence of others). Yet, no one reported using the mechanisms to avoid information leaks, which suggests that the typical mechanisms available for controlling the device state might not be adequate to mitigate the information disclosure risks posed by notifications. Along with reasons connected to privacy, participants reported using devicecontrol mechanisms for other reasons such as preserving battery life, restarting the device, driving, eating, or special circumstances.

We then tried to understand whether the use of various devicecontrol mechanisms is associated with negative experiences connected to notifications. To this end, we grouped the responses based on the number of negative experiences and calculated the group-wise mean usage (in hours) in a typical day (see Table 4). Kruskal-Wallis tests showed statistically significant differences in the use of the Do Not Disturb mode $(p<0.05)$ and notification management apps $(p<0.05)$ based on the number of negative experiences. Notably, the use of device-control mechanisms seems to increase until ten instances of negative experiences, but decreases thereafter.

We calculated the correlation between the hourly use of each device-control mechanism and the number of negative experiences (see Table 4), adjusting the p values using Bonferroni correction. We found that the number of negative experiences is positively correlated with the use of the Do Not Disturb mode, switchedoff mode, airplane mode, and notification management apps $(p<$ $0.001)$. 
Table 3: Mean scores for the Perceived Intrusion and Perceived Surveillance constructs from the MUIPC scale on a sevenpoint scale (higher scores correspond to greater concern) and mean use of various device-control mechanisms (in hours/day), grouped by number of negative experiences connected to notifications.

\begin{tabular}{|c|c|c|c|c|c|c|c|}
\hline \multirow[b]{2}{*}{$\begin{array}{l}\text { Negative } \\
\text { Experiences }\end{array}$} & \multicolumn{2}{|c|}{ MUIPC } & \multicolumn{4}{|c|}{ Device-control Mechanisms } & \multirow[b]{2}{*}{$\begin{array}{c}\text { Notification } \\
\text { Apps }\end{array}$} \\
\hline & $\begin{array}{l}\text { Perceived } \\
\text { Intrusion }\end{array}$ & $\begin{array}{c}\text { Perceived } \\
\text { Surveillance }\end{array}$ & $\begin{array}{l}\text { DND } \\
\text { Mode }\end{array}$ & $\begin{array}{l}\text { Silent } \\
\text { Mode }\end{array}$ & $\begin{array}{l}\text { Device } \\
\text { Off }\end{array}$ & $\begin{array}{c}\text { Airplane } \\
\text { Mode }\end{array}$ & \\
\hline 0 & 4.87 & 4.97 & 1.64 & 6.11 & 1.05 & 1.08 & 2.18 \\
\hline $1-2$ & 5.14 & 5.18 & 2.85 & 5.58 & 1.50 & 1.42 & 4.88 \\
\hline $3-5$ & 5.29 & 5.36 & 3.02 & 6.28 & 2.96 & 2.89 & 5.72 \\
\hline $6-10$ & 5.36 & 5.50 & 6.64 & 9.43 & 6.21 & 8.21 & 10.43 \\
\hline $11-20$ & 6.11 & 5.67 & 7.00 & 6.00 & 5.33 & 6.00 & 4.33 \\
\hline $20+$ & 6.00 & 5.87 & 2.20 & 4.20 & 0.40 & 0.20 & 2.00 \\
\hline
\end{tabular}

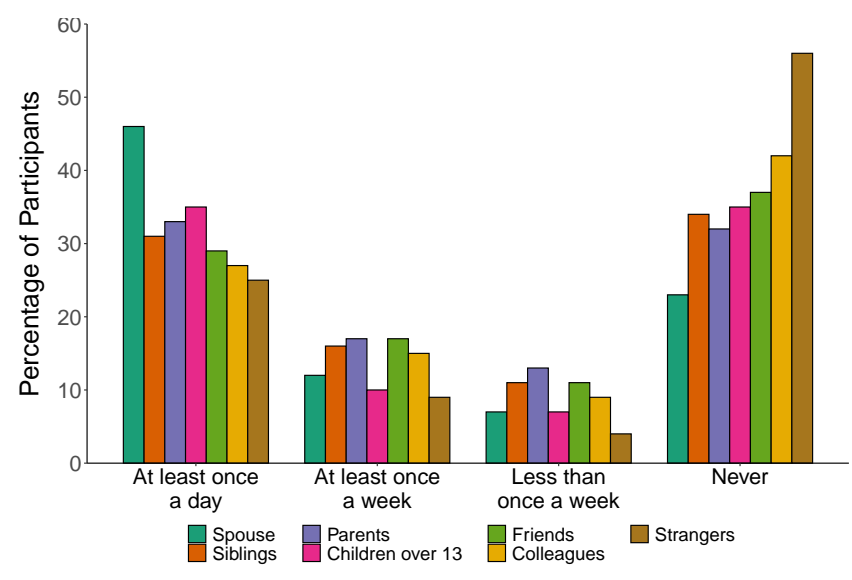

(a) Frequency of device sharing with various parties.

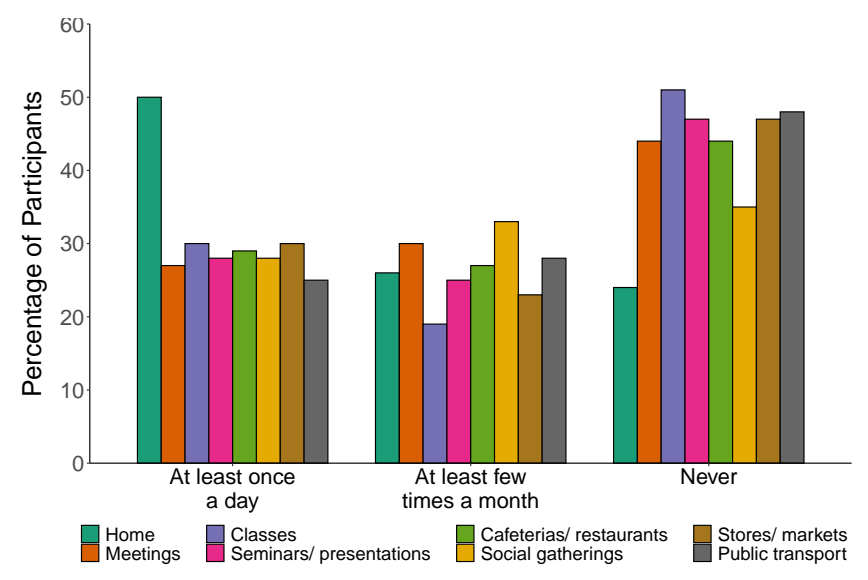

(b) Frequency for others viewing notifications in various settings.

Figure 4: Device sharing and undesired exposure of other people's information.

Table 4: Mean use of mechanisms to control the device state (in hours/day) and correlation between the use of the devicecontrol mechanisms and the number of negative experiences connected to notifications.

\begin{tabular}{lcc}
\hline $\begin{array}{l}\text { Device-control } \\
\text { Mechanism }\end{array}$ & $\begin{array}{c}\text { Mean } \\
\text { (hours/day) }\end{array}$ & $\begin{array}{c}\text { Correlation with } \\
\text { Negative } \\
\text { Experiences }\end{array}$ \\
\hline DND mode & 2.80 & $0.31^{* * *}$ \\
Silent mode & 6.20 & 0.14 \\
Device off & 2.12 & $0.31^{* * *}$ \\
Airplane mode & 2.24 & $0.32^{* * *}$ \\
Notification apps & 4.51 & $0.38^{* * *}$ \\
\hline & $* * *: p<0.001$ with Bonferroni correction
\end{tabular}

Digital Difficulties. We used the General Digital Difficulties subscale of the Digital Difficulties scale [6] as a measure of technical proficiency. Table 6 provides the mean scores of the participants on the General Digital Difficulties subscale, grouped by the number of negative experiences. Higher scores indicate greater general digital difficulties, i.e., lower technical proficiency. Interestingly, we found that those who indicated higher technical proficiency reported either no negative experiences with notifications at all or a large number (more than 10) of negative experiences. Compared to these two groups of participants at each end, those who reported 1-10 instances of notification-related negative experiences were less technically proficient.

\section{DISCUSSION}

Our findings show that privacy concerns related to smartphone notifications are influenced by the information content and additional audiences beyond the receiver, thus echoing privacy-related findings from the other domains covered in the literature (see Section 2). At the same time, we extend the literature by adding notificationspecific nuance and surfacing additional privacy-affecting aspects connected to notifications. For instance, we found that privacy concerns for notifications vary based on the type of app. In future work, it would be interesting to investigate if users further differentiate 


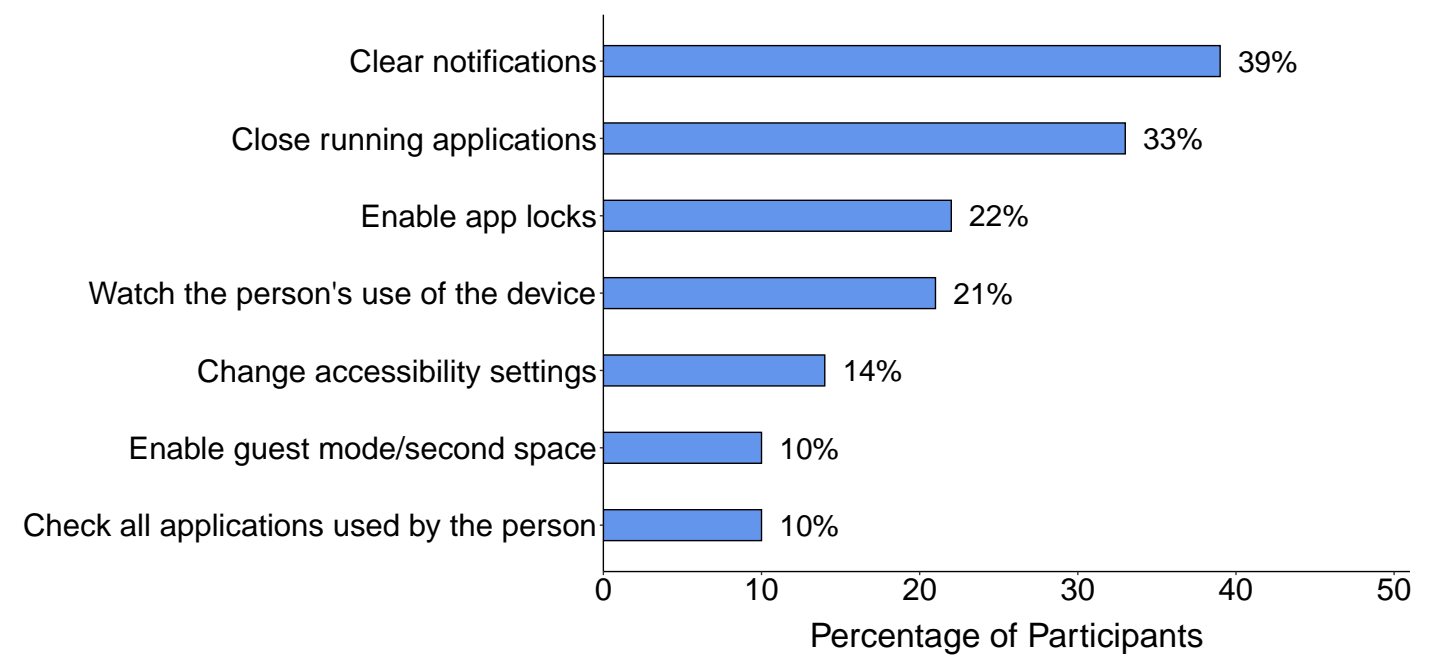

Figure 5: Actions taken when sharing a device with other parties.

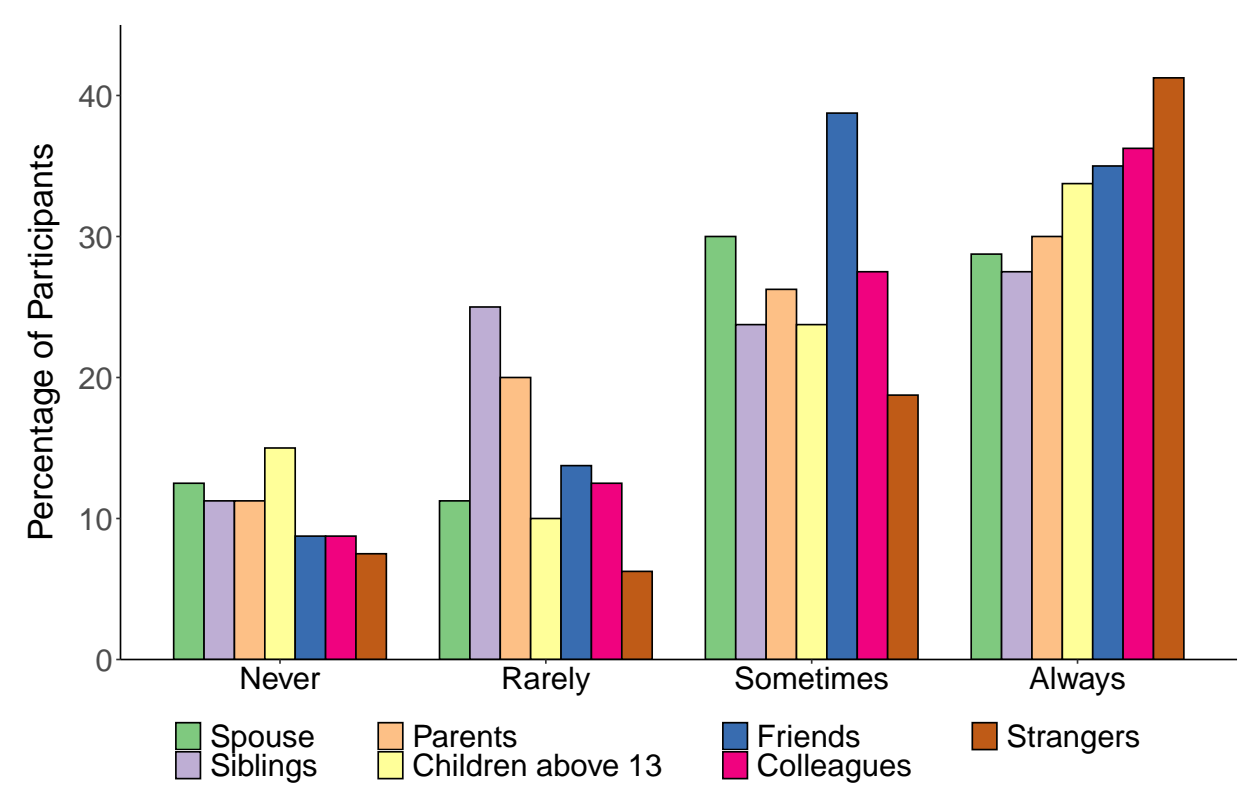

Figure 6: Frequency of clearing notifications prior to handing the device over to various parties.

privacy sensitivity of notifications from a single app that delivers different types of notifications for different features within the app (e.g., messaging apps typically permit users to set different types of notification settings for different types of events within the apps).

Apart from notification content, we note that the disruption caused by notification alerts (such as sounds) impinges upon the Solitude facet of privacy [47]. Participants reported deleting apps that send notifications too frequently. Further, the content of the notifications can impact the Intimacy and Reserve facets of privacy [47] by causing misunderstanding, unease, and conflict that have the potential for a negative impact on interpersonal relationships. Yet, current mechanisms for controlling notification delivery do not provide any consideration for the user's social context at the time of notification delivery. While previous studies to predict opportune moments of notification delivery (e.g., $[17,35])$ have shown that delaying notification can partially reduce interruptibility (the intrusion facet of privacy), it would be of interest to examine if notification deferral can mitigate other types of privacy violations as well. Notifications that contain undesirable or malicious content, such as spam, advertisements, malicious links, etc., can raise the privacy issue of digital intrusion [40]. While infrastructure and end-point solutions exist for detecting and filtering email spam, there are no analogous mechanisms for notification content. 
Table 5: Reasons behind preferences for controlling the device state.

\begin{tabular}{|c|c|c|c|}
\hline Theme & Code & Count & Example Comments \\
\hline \multirow{7}{*}{$\begin{array}{l}\text { Prefer to } \\
\text { control }\end{array}$} & Avoid sounds & 6 & $\begin{array}{l}\text { "Silent mode is the best with a 'shake' alert. In this } \\
\text { case I'm notified 'minimally' without the dreaded sound } \\
\text { going off." (P138) }\end{array}$ \\
\hline & Limit interruptions & 4 & $\begin{array}{l}\text { "I keep my phone in silent mode at all times. I find this } \\
\text { works best for me and limits distractions." (P47) }\end{array}$ \\
\hline & Prevent disturbance of sleep & 17 & $\begin{array}{l}\text { "I turn off my phone when I go to sleep and turn it back } \\
\text { on when I wake up." (P136) }\end{array}$ \\
\hline & $\begin{array}{l}\text { Focus during work or specific } \\
\text { tasks }\end{array}$ & 17 & $\begin{array}{l}\text { "I make sure I can't be disturbed at all when I'm work- } \\
\text { ing. It's just easier to turn on DND than fiddle with } \\
\text { individual apps." (P147) }\end{array}$ \\
\hline & $\begin{array}{l}\text { Interact with others (meet- } \\
\text { ings, social gathering, etc.) }\end{array}$ & 6 & $\begin{array}{l}\text { "I generally have my phone on silent for at least about } \\
4 \text { hours in the evening when spending time with the } \\
\text { family." (P10) }\end{array}$ \\
\hline & Customize settings & 4 & $\begin{array}{l}\text { "I just set custom individual notification settings for } \\
\text { most of my apps. If they're atrociously annoying, I unin- } \\
\text { stall them." (P51) }\end{array}$ \\
\hline & Other reasons & 5 & $\begin{array}{l}\text { "During office hours, I use Do Not Disturb mode. Device } \\
\text { is off while eating lunch." (P157) }\end{array}$ \\
\hline \multirow{3}{*}{$\begin{array}{l}\text { Do not } \\
\text { control }\end{array}$} & Be available for emergencies & 2 & $\begin{array}{l}\text { "I keep it [notifications] on just in case there's an emer- } \\
\text { gency and someone is trying to reach me." (P106) }\end{array}$ \\
\hline & Avoid missing alerts & 4 & $\begin{array}{l}\text { "I prefer to get all alerts and notifications when they } \\
\text { arrive, regardless of the time of the day." (P103) }\end{array}$ \\
\hline & Other reasons & 7 & $\begin{array}{l}\text { "Since I live alone and I usually do not get late calls, I } \\
\text { leave the cell phone on." (P57) }\end{array}$ \\
\hline
\end{tabular}

Table 6: Mean Digital Difficulties scores grouped by the number of negative experiences connected to notifications. Higher difficulty scores indicate lower technical efficacy.

\begin{tabular}{cccc}
\hline $\begin{array}{c}\text { Negative } \\
\text { Experiences }\end{array}$ & Mean & Median & SD \\
\hline 0 & 2.12 & 1.6 & 1.30 \\
$1-2$ & 3.15 & 3.6 & 1.31 \\
$3-5$ & 3.10 & 3.6 & 1.24 \\
$6-10$ & 3.53 & 3.8 & 0.95 \\
$11-20$ & 2.47 & 1.8 & 1.89 \\
$20+$ & 2.36 & 1.6 & 1.62 \\
\hline
\end{tabular}

As reported by past studies on device sharing, the practices of the participants in our study confirm that users do share their devices with others, even strangers, or engage in joint use of their devices, such as watching a video with someone. While current smartphones offer the privacy-protecting option of using a 'guest' mode when sharing a device, such all-or-none binary access control is neither adequate nor well-suited to handle the variety of situations under which device sharing occurs [20]. It should be no surprise then that we found little use of these mechanisms. Instead, to avoid privacy leaks from notifications during device sharing, many participants opted for crude-but-quick solutions such as clearing notifications or closing apps, and many did not take any precautions at all. Apart from poor usability and user experience, additional factors behind the low use of such privacy-protective features might be: forgetting to activate the feature, avoiding the social awkwardness of signaling distrust by overt and obvious use of the feature, needing access to specific functionality or data that is not available in a restricted guest mode, or fearing the loss of important notifications. As a participant reported, "sometimes I clear the important notifications [before sharing the device] then I forget to answer" (P202). Further research is needed to develop mechanisms that can facilitate a seamless and socially workable solution for transition to a mode that protects notification privacy in contexts of shared device use.

Even when not sharing the device, we found that people do not take advantage of the more sophisticated options for controlling notification delivery that are available within smartphone operating systems and apps, instead relying mostly on simplistic mechanisms such as silent or DND modes. The use of these modes does mean that the user can miss out on important calls or urgent messages. At the same time, slightly more participants reported the use of notification management apps (62\%) than the use of the DND mode $(56 \%)$. Given that a large percentage of smartphone users report using third-party apps to control notifications, it would be interesting to understand the advantages these apps offer over the notificationcontrol mechanisms provided by the operating systems.

Although the latest versions of smartphone operating systems include greater control over notifications, these features do not cover all of the privacy issues surfaced by our findings. For instance, iOS allows users to customize lock-screen notification previews 
by app [13]. However, these options cannot be tailored based on social or temporal contexts. Moreover, when an unlocked device is shared with someone else, notifications can leak information via the notification center and/or pop-up alerts (if the user has enabled the option to show notification previews when the device is not locked). The recent feature for snoozing notifications ${ }^{1}$ introduced in Android versions 8 and above might not be helpful in preventing information leaks either because the information might be exposed when notifications are redelivered later. While the DND mode in Android versions 9 and above can be helpful for limiting interruptions from notifications, ${ }^{2}$ Android users in our sample did not report using the feature to limit risks of information disclosure. Additionally, the latest version of Android includes 'digital well-being' features [11] such as a 'Focus Mode' and allows users to flip the phone down to turn notifications off. While these recent features can be helpful in reducing distractions and interruptions from notifications, they do not address the various other privacy issues related to notifications uncovered by the findings of our study. For instance, none of the features provides a means for users to disable notifications while device sharing or screencasting.

While those who encounter a few negative experiences seem to increase their use of device-control options, the tendency appears to reverse after a handful of such experiences, with those reporting large numbers of negative experiences also reporting lower usage of device-control options, despite being technically proficient. Further research is needed to investigate whether these findings are driven by a lack of effective ways to scale the control mechanisms to handle a large volume of potentially privacy-affecting notifications or stem from user acceptance of negative experiences with notifications as routine part of everyday device use.

\section{IMPLICATIONS}

Our findings suggest a number of design improvements to help users avoid privacy violations connected to notifications received on their mobile devices.

\subsection{Personalize Notification Content and Delivery Frequency}

As a participant reported, "Group chats are always annoying, and they send a lot of notifications. It is distracting and irritating especially when you're busy. Also, some of the apps frequently send notifications, and it's frustrating that I can not customize them." (P34). We recommend that app developers allow users to opt in for notifications for only the most preferred content from the app. In this regard, the recent versions of the Android operating system (Android 8.0 and above) provide greater control over app notifications through the mechanism of 'channels,' which are content categories that can be individually turned on or off. For example, a social media app could create separate 'notification channels' for different content, such as group messages, direct messages, mentions, likes, follows, etc. [16].

Recently, Weber et al. [43] investigated the reasons behind manual notification deferment. We recommend studies to understand if deferral of notifications might be helpful in reducing privacy risks

\footnotetext{
${ }^{1}$ https://support.google.com/android/answer/9079661

${ }^{2}$ https://support.google.com/android/answer/9069335
}

or avoiding privacy breaches. Further, mechanisms for opportune delivery can be extended for considerations beyond interruptiblity to include other privacy-affecting contextual factors, such as the presence of other parties who can view the screen, use of the device by someone other than the owner, etc. For example, the 'Hide sensitive content' option for lock-screen notifications in Android could be extended to be location-dependent.

\subsection{Protect Notification Privacy During Device Sharing}

Our study reveals that notifications can cause unintended information disclosure when the device is shared with others. Therefore, we recommend automatically turning notifications off when it is detected that the device is handed over to someone else, device screen is cast to a publicly viewable display, or multiple people are watching the device screen. The detection of such 'public' contexts can be based on explicit user action and/or relevant heuristics and/or machine learning techniques. For instance, machine learning algorithms could be trained for automatic detection of contexts of shared use and additional audiences beyond the device owner based on features such as the characteristics of the person handling the device, temporal factors (e.g., business hours vs. sleep time), sensor readings of the environmental context [29], etc. For additional protection, users can be provided the ability to assign password protection for accessing the notification collection (i.e., the notification drawer or notification center) [45]. Such password protection will prevent the other party from accessing notifications from the collection so that users do not need to clear them prior to sharing the device with someone else and risk losing important notifications as a result.

Further, natural language processing (NLP) algorithms could be employed on the device to examine notification content and prevent risks of information disclosure for parties other than the recipient. For instance, NLP could help detect and obfuscate people's names when displaying notifications in a shared context. As a study by Weber et al. [46] shows, users chose to preserve the privacy of their contacts by censoring names before logging notification content, thus underscoring the utility of automating such obfuscation.

\subsection{Limit the Number of Notifications}

Several participants reported feeling uneasy and uncomfortable due to the intrusion caused by notifications. High frequency of notifications caused participants to experience negative emotions, such as annoyance and irritation. Therefore, limiting the number of notifications can decrease digital intrusion from unwanted content, reduce disruption of solitude, and increase the overall user experience of notifications. Moreover, lowering notification frequency reduces the number of occasions for potential privacy violation, thus reducing privacy risk. We recommend limiting the number of notifications in one or more of the following ways:

(1) Allow users to control notification delivery based on specified keywords, such as urgent, OTP, update, important.

(2) Bundle notifications and display the entire collection only upon reaching a threshold number of notifications or at set time intervals, such as every hour, or at fixed times of the day, say noon. 
(3) Permit users to set per-app limits on the number of notifications within a given time interval.

\section{LIMITATIONS AND FUTURE WORK}

Our findings are impacted by the limitations of self-selection and self-reporting. Future work should compare these results with realworld analytics of behavioral data and metadata that captures notification content and user interactions with notifications via tools such as Clear All [45] or Notification Log [44]. Further, tools like Annotif [46] can enable the collection of corresponding retrospective user reflection that adds context to the captured logs and facilitates a more nuanced interpretation based on a combination of analytics and user responses.

Our sample consists of individuals from the United States. Since privacy is known to be influenced by cultural factors, generalizability to other cultures requires verification and is an important direction for future work. Apart from privacy concerns, device sharing practices in particular are likely to differ substantially in developing nations because of various sociocultural differences.

Our investigation focused on mobile devices (smartphones, in particular). It would be interesting to compare these results with privacy aspects of notifications from other smart devices such as those embedded in a user's physical environment (i.e., Internet of Things [IoT] devices).

\section{CONCLUSION}

Users of mobile devices typically receive dozens of notifications every day. We found that inopportune notifications can result in undesirable information disclosure and disruption, thus violating privacy. The portable nature of mobile devices exacerbates these aspects because the devices are often used in situations in which other people are around and can view the contents on the device screen. Moreover, the devices can be easily shared with other people by simply handing them over. Yet, preference settings to control mobile-device notifications currently lack any consideration of such usage contexts with high potential for privacy violation. The findings of our study highlight the need for greater attention to control over notification delivery in order to avoid privacy violations for the user as well as other parties whose information might be present in the notification content.

\section{ACKNOWLEDGMENTS}

We thank the participants of the study. We acknowledge Morgan L. Brockman and Nicholas Micallef for coding the qualitative responses. Thanks are due to Sadia Khan, Xinyao Ma, Dalyapraz Manatova, John S. Seberger, Emily Swiatek, and Ying Tang for their help in piloting and improving the questionnaire. We are grateful for the feedback of anonymous reviewers that helped improve the paper.

\section{REFERENCES}

[1] Syed Ishtiaque Ahmed, Md. Romael Haque, Jay Chen, and Nicola Dell. 2017 Digital Privacy Challenges with Shared Mobile Phone Use in Bangladesh. Proc ACM Hum.-Comput. Interact. 1, CSCW, Article 17 (Dec. 2017), 20 pages. https: //doi.org/10.1145/3134652

[2] Mohammed Eunus Ali, Anika Anwar, Ishrat Ahmed, Tanzima Hashem, Lars Kulik, and Egemen Tanin. 2014. Protecting Mobile Users from Visual Privacy Attacks. In Proceedings of the 2014 ACM International foint Conference on Pervasive and Ubiquitous Computing: Adjunct Publication (Seattle, Washington) (UbiComp '14 Adjunct). Association for Computing Machinery, New York, NY, USA, 1-4. https://doi.org/10.1145/2638728.2638788

[3] Hunt Allcott and Matthew Gentzkow. 2017. Social Media and Fake News in the 2016 Election. Journal of Economic Perspectives 31, 2 (May 2017), 211-236. https://doi.org/10.1257/jep.31.2.211

[4] Hazim Almuhimedi, Florian Schaub, Norman Sadeh, Idris Adjerid, Alessandro Acquisti, Joshua Gluck, Lorrie Faith Cranor, and Yuvraj Agarwal. 2015. Your Location Has Been Shared 5,398 Times! A Field Study on Mobile App Privacy Nudging. In Proceedings of the 33rd Annual ACM Conference on Human Factors in Computing Systems (CHI '15). Association for Computing Machinery, New York, NY, USA, 787-796. https://doi.org/10.1145/2702123.2702210

[5] Irwin Altman. 1975. The Environment and Social Behavior: Privacy, Personal Space, Territory, Crowding. Brooks/Cole Publishing Company.

[6] Sarah Anrijs, Koen Ponnet, and Lieven De Marez. 2020. Development and Psychometric Properties of the Digital Difficulties Scale (DDS): An Instrument to Measure who is Disadvantaged to Fulfill Basic Needs by Experiencing Difficulties in Using a Smartphone or Computer. PLOS ONE 15, 5 (2020), e0233891. https://doi.org/10.1371/journal.pone.0233891

[7] Rebecca Balebako, Jaeyeon Jung, Wei Lu, Lorrie Faith Cranor, and Carolyn Nguyen. 2013. "Little Brothers Watching You": Raising Awareness of Data Leaks on Smartphones. In Proceedings of the Ninth Symposium on Usable Privacy and Security (Newcastle, United Kingdom) (SOUPS '13). Association for Computing Machinery, New York, NY, USA, Article 12, 11 pages. https: //doi.org/10.1145/2501604.2501616

[8] Rebecca Balebako, Pedro G. Leon, Hazim Almuhimedi, Patrick Gage Kelley, Jonathan Mugan, Alessandro Acquisti, Lorrie Cranor, and Norman SadehKoniecpol. 2011. Nudging Users Towards Privacy on Mobile Devices. In Proceedings of the CHI 2011 Workshop on Persuasion, Nudge, Influence and Coercion. 4 pages.

[9] Antoine Boutet and Sébastien Gambs. 2019. Inspect What Your Location History Reveals About You: Raising User Awareness on Privacy Threats Associated with Disclosing His Location Data. In Proceedings of the 28th ACM International Conference on Information and Knowledge Management (Beijing, China) (CIKM '19). Association for Computing Machinery, New York, NY, USA, 2861-2864. https://doi.org/10.1145/3357384.3357837

[10] Virginia Braun, Victoria Clarke, Nikki Hayfield, and Gareth Terry. 2019. Thematic Analysis. In Handbook of Research Methods in Health Social Sciences, Pranee Liamputtong (Ed.). Springer Singapore, Singapore, 843-860. https://doi.org/10. 1007/978-981-10-5251-4 103

[11] Sydney Butler. 2021. What is Digital Wellbeing for Android and How to Use It. https://www.online-tech-tips.com/smartphones/what-is-digital-wellbeingfor-android-and-how-to-use-it/ Accessed: 2021-06-30.

[12] Hyunsung Cho, Jinyoung Oh, Juho Kim, and Sung-Ju Lee. 2020. I Share, You Care: Private Status Sharing and Sender-Controlled Notifications in Mobile Instant Messaging. Proc. ACM Hum.-Comput. Interact. 4, CSCW1, Article 034 (May 2020), 25 pages. https://doi.org/10.1145/3392839

[13] Jason Cross. 2018. iOS 11: How to Hide Sensitive Info in Notification Previews. https://www.macworld.com/article/231076/ios-11-how-to-hidesensitive-info-in-notification-previews.html Accessed: 2021-06-30.

[14] Alexander De Luca, Marian Harbach, Emanuel von Zezschwitz, Max-Emanuel Maurer, Bernhard Ewald Slawik, Heinrich Hussmann, and Matthew Smith. 2014. Now You See Me, Now You Don't: Protecting Smartphone Authentication from Shoulder Surfers. In Proceedings of the SIGCHI Conference on Human Factors in Computing Systems (Toronto, Ontario, Canada) (CHI '14). Association for Computing Machinery, New York, NY, USA, 2937-2946. https://doi.org/10.1145/ 2556288.2557097

[15] Malin Eiband, Mohamed Khamis, Emanuel von Zezschwitz, Heinrich Hussmann, and Florian Alt. 2017. Understanding Shoulder Surfing in the Wild: Stories from Users and Observers. In Proceedings of the 2017 CHI Conference on Human Factors in Computing Systems (CHI '17). Association for Computing Machinery, New York, NY, USA, 4254-4265. https://doi.org/10.1145/3025453.3025636

[16] Joe Fedewa. 2021. What Are Android Notification Channels? https: //www.howtogeek.com/715614/what-are-android-notification-channels/ Accessed: 2021-06-30.

[17] Joel E. Fischer, Chris Greenhalgh, and Steve Benford. 2011. Investigating Episodes of Mobile Phone Activity as Indicators of Opportune Moments to Deliver Notifications. In Proceedings of the 13th International Conference on Human Computer Interaction with Mobile Devices and Services (Stockholm, Sweden) (MobileHCI '11). Association for Computing Machinery, New York, NY, USA, 181-190. https://doi.org/10.1145/2037373.2037402

[18] Jan Gugenheimer, Alexander De Luca, Hayato Hess, Stefan Karg, Dennis Wolf, and Enrico Rukzio. 2015. ColorSnakes: Using Colored Decoys to Secure Authentication in Sensitive Contexts. In Proceedings of the 17th International Conference on Human-Computer Interaction with Mobile Devices and Services (Copenhagen, Denmark) (MobileHCI '15). Association for Computing Machinery, New York, NY, USA, 274-283. https://doi.org/10.1145/2785830.2785834 
[19] Alina Hang, Emanuel von Zezschwitz, Alexander De Luca, and Heinrich Hussmann. 2012. Too Much Information! User Attitudes Towards Smartphone Sharing. In Proceedings of the 7th Nordic Conference on Human-Computer Interaction: Making Sense Through Design (Copenhagen, Denmark) (NordiCHI '12). Association for Computing Machinery, New York, NY, USA, 284-287. https://doi.org/10.1145/2399016.2399061

[20] Amy K. Karlson, A.J. Bernheim Brush, and Stuart Schechter. 2009. Can I Borrow Your Phone? Understanding Concerns When Sharing Mobile Phones. In Proceedings of the SIGCHI Conference on Human Factors in Computing Systems (CHI '09). Association for Computing Machinery, New York, NY, USA, 1647-1650. https://doi.org/10.1145/1518701.1518953

[21] Yongsung Kim, Adam Fourney, and Ece Kamar. 2019. Studying Preferences and Concerns about Information Disclosure in Email Notifications. In The World Wide Web Conference (San Francisco, CA, USA) (WWW' 19). Association for Computing Machinery, New York, NY, USA, 874-885. https://doi.org/10.1145/ 3308558.3313451

[22] Alfred Kobsa, Sameer Patil, and Bertolt Meyer. 2012. Privacy in Instant Messaging: An Impression Management Model. Behaviour \& Information Technology 31, 4 (2012), 355-370. https://doi.org/10.1080/01449291003611326

[23] Nicole C. Krämer and Nina Haferkamp. 2011. Online Self-Presentation: Balancing Privacy Concerns and Impression Construction on Social Networking Sites. In Privacy Online: Perspectives on Privacy and Self-Disclosure in the Social Web, Sabine Trepte and Leonard Reinecke (Eds.). Springer Berlin Heidelberg, Berlin, Heidelberg, 127-141. https://doi.org/10.1007/978-3-642-21521-6_10

[24] Naresh K. Malhotra, Sung S. Kim, and James Agarwal. 2004. Internet Users' Information Privacy Concerns (IUIPC): The Construct, the Scale, and a Causal Model. Information Systems Research 15, 4 (2004), 336-355. https://doi.org/10. 1287/isre.1040.0032

[25] Stephen T. Margulis. 2011. Three Theories of Privacy: An Overview. In Privacy Online: Perspectives on Privacy and Self-Disclosure in the Social Web, Sabine Trepte and Leonard Reinecke (Eds.). Springer Berlin Heidelberg, Berlin, Heidelberg, 9-17. https://doi.org/10.1007/978-3-642-21521-6_2

[26] Tara Matthews, Kerwell Liao, Anna Turner, Marianne Berkovich, Robert Reeder, and Sunny Consolvo. 2016. "She'll Just Grab Any Device That's Closer": A Study of Everyday Device \& Account Sharing in Households. In Proceedings of the 2016 CHI Conference on Human Factors in Computing Systems (CHI '16). Association for Computing Machinery, New York, NY, USA, 5921-5932. https: //doi.org/10.1145/2858036.2858051

[27] Abhinav Mehrotra, Mirco Musolesi, Robert Hendley, and Veljko Pejovic. 2015 Designing Content-Driven Intelligent Notification Mechanisms for Mobile Applications. In Proceedings of the 2015 ACM International foint Conference on Pervasive and Ubiquitous Computing (Osaka, Japan) (UbiComp '15). Association for Computing Machinery, New York, NY, USA, 813-824. https://doi.org/10.1145/2750858. 2807544

[28] Nicholas Micallef, Mike Just, Lynne Baillie, and Maher Alharby. 2017. Stop Annoying Me! An Empirical Investigation of the Usability of App Privacy Notifications. In Proceedings of the 29th Australian Conference on Computer-Human Interaction (Brisbane, Queensland, Australia) (OZCHI '17). Association for Computing Machinery, New York, NY, USA, 371-375. https://doi.org/10.1145/3152771.3156139

[29] Nicholas Micallef, Mike Just, Lynne Baillie, Martin Halvey, and Hilmi Güneş Kayacik. 2015. Why Aren't Users Using Protection? Investigating the Usability of Smartphone Locking. In Proceedings of the 17th International Conference on Human-Computer Interaction with Mobile Devices and Services (Copenhagen, Denmark) (MobileHCI '15). Association for Computing Machinery, New York, NY, USA, 284-294. https://doi.org/10.1145/2785830.2785835

[30] Helen Nissenbaum. 2004. Privacy as Contextual Integrity. Washington Law Review 79 (2004), 119-158.

[31] Judith S. Olson, Jonathan Grudin, and Eric Horvitz. 2005. A Study of Preferences for Sharing and Privacy. In CHI'05 Extended Abstracts on Human Factors in Computing Systems (CHI '05). Association for Computing Machinery, New York, NY, USA, 1985-1988. https://doi.org/10.1145/1056808.1057073

[32] Sameer Patil and Jennifer Lai. 2005. Who Gets to Know What When: Configuring Privacy Permissions in an Awareness Application. In Proceedings of the SIGCHI Conference on Human Factors in Computing Systems (CHI '04). Association for Computing Machinery, New York, NY, USA, 101-110. https://doi.org/10.1145/ 1054972.1054987

[33] Sameer Patil, Greg Norcie, Apu Kapadia, and Adam J. Lee. 2012. Reasons, Rewards, Regrets: Privacy Considerations in Location Sharing as an Interactive Practice. In Proceedings of the Eighth Symposium on Usable Privacy and Security (Washington, D.C.) (SOUPS '12). Association for Computing Machinery, New York, NY, USA, Article 5, 15 pages. https://doi.org/10.1145/2335356.2335363

[34] Veljko Pejovic and Mirco Musolesi. 2014. InterruptMe: Designing Intelligent Prompting Mechanisms for Pervasive Applications. In Proceedings of the 2014 ACM International foint Conference on Pervasive and Ubiquitous Computing (Seattle, Washington) (UbiComp '14). Association for Computing Machinery, New
York, NY, USA, 897-908. https://doi.org/10.1145/2632048.2632062

[35] Martin Pielot, Bruno Cardoso, Kleomenis Katevas, Joan Serrà, Aleksandar Matic, and Nuria Oliver. 2017. Beyond Interruptibility: Predicting Opportune Moments to Engage Mobile Phone Users. Proc. ACM Interact. Mob. Wearable Ubiquitous Technol. 1, 3, Article 91 (Sept. 2017), 25 pages. https://doi.org/10.1145/3130956

[36] Martin Pielot, Karen Church, and Rodrigo de Oliveira. 2014. An In-Situ Study of Mobile Phone Notifications. In Proceedings of the 16th International Conference on Human-Computer Interaction with Mobile Devices \& Services (Toronto, ON, Canada) (MobileHCI '14). Association for Computing Machinery, New York, NY, USA, 233-242. https://doi.org/10.1145/2628363.2628364

[37] Martin Pielot, Amalia Vradi, and Souneil Park. 2018. Dismissed! A Detailed Exploration of How Mobile Phone Users Handle Push Notifications. In Proceedings of the 20th International Conference on Human-Computer Interaction with Mobile Devices and Services (Barcelona, Spain) (MobileHCI '18). Association for Computing Machinery, New York, NY, USA, Article 3, 11 pages. https://doi.org/10.1145/3229434.3229445

[38] Oriana Riva, Chuan Qin, Karin Strauss, and Dimitrios Lymberopoulos. 2012. Progressive Authentication: Deciding When to Authenticate on Mobile Phones. In 21st USENIX Security Symposium (USENIX Security 12). USENIX Association, Bellevue, WA, 301-316. https://www.usenix.org/conference/usenixsecurity12/ technical-sessions/presentation/riva

[39] H. Jeff Smith and Sandra J. Milberg. 1996. Information Privacy: Measuring Individuals' Concerns about Organizational Practices. MIS Q. 20, 2 (June 1996), 167-196. https://doi.org/10.2307/249477

[40] Daniel J. Solove. 2006. A Taxonomy of Privacy. University of Pennsylvania Law Review 154, 3 (2006), 477-560.

[41] Raj Vardhan, Ameya Sanzgiri, Dattatraya Kulkarni, Piyush Joshi, and Srikanth Nalluri. 2017. Notify Assist: Balancing Privacy and Convenience in Delivery of Notifications on Android Smartphones. In Proceedings of the 2017 on Workshop on Privacy in the Electronic Society (Dallas, Texas, USA) (WPES '17). Association for Computing Machinery, New York, NY, USA, 17-20. https://doi.org/10.1145/ 3139550.3139561

[42] Emanuel von Zezschwitz, Sigrid Ebbinghaus, Heinrich Hussmann, and Alexander De Luca. 2016. You Can't Watch This! Privacy-Respectful Photo Browsing on Smartphones. In Proceedings of the 2016 CHI Conference on Human Factors in Computing Systems (CHI '16). Association for Computing Machinery, New York, NY, USA, 4320-4324. https://doi.org/10.1145/2858036.2858120

[43] Dominik Weber, Alexandra Voit, Jonas Auda, Stefan Schneegass, and Niels Henze. 2018. Snooze! Investigating the User-Defined Deferral of Mobile Notifications. In Proceedings of the 20th International Conference on Human-Computer Interaction with Mobile Devices and Services (Barcelona, Spain) (MobileHCI '18). Association for Computing Machinery, New York, NY, USA, Article 2, 13 pages. https: //doi.org/10.1145/3229434.3229436

[44] Dominik Weber, Alexandra Voit, and Niels Henze. 2018. Notification Log: An Open-Source Framework for Notification Research on Mobile Devices. In Proceedings of the 2018 ACM International foint Conference and 2018 International Symposium on Pervasive and Ubiquitous Computing and Wearable Computers (Singapore, Singapore) (UbiComp '18). Association for Computing Machinery, New York, NY, USA, 1271-1278. https://doi.org/10.1145/3267305.3274118

[45] Dominik Weber, Alexandra Voit, and Niels Henze. 2019. Clear All: A LargeScale Observational Study on Mobile Notification Drawers. In Proceedings of Mensch Und Computer 2019 (Hamburg, Germany) (MuC '19). Association for Computing Machinery, New York, NY, USA, 361-372. https://doi.org/10.1145/ 3340764.3340765

[46] Dominik Weber, Alexandra Voit, Gisela Kollotzek, and Niels Henze. 2019. Annotif: A System for Annotating Mobile Notifcations in User Studies. In Proceedings of the 18th International Conference on Mobile and Ubiquitous Multimedia (Pisa, Italy) (MUM '19). Association for Computing Machinery, New York, NY, USA, Article 24, 12 pages. https://doi.org/10.1145/3365610.3365611

[47] Alan F. Westin. 1967. Privacy and Freedom. Atheneum.

[48] Pamela Wisniewski, A. K. M. Najmul Islam, Bart P. Knijnenburg, and Sameer Patil. 2015. Give Social Network Users the Privacy They Want. In Proceedings of the 18th ACM Conference on Computer Supported Cooperative Work \& Social Computing (Vancouver, BC, Canada) (CSCW' 15). Association for Computing Machinery, New York, NY, USA, 1427-1441. https://doi.org/10.1145/2675133.2675256

[49] Heng Xu, Sumeet Gupta, Mary Beth Rosson, and John M. Carroll. 2012. Measuring Mobile Users' Concerns for Information Privacy. In Thirty Third International Conference on Information Systems (ICIS 2012, Vol. 3). Association for Information Systems, Orlando, FL, USA, 2278-2293.

[50] Fengpeng Yuan, Xianyi Gao, and Janne Lindqvist. 2017. How Busy Are You? Predicting the Interruptibility Intensity of Mobile Users. In Proceedings of the 2017 CHI Conference on Human Factors in Computing Systems (CHI '17). Association for Computing Machinery, New York, NY, USA, 5346-5360. https://doi.org/10. $1145 / 3025453.3025946$ 